\title{
Metodologia Alternativa para Resolução do Modelo de Difusão de Rakhmatov-Vrudhula Aplicada à Modelagem Matemática do Tempo de Vida de Baterias Utilizadas em Dispositivos Móveis
}

\author{
Douglas Joziel Bitencourt Freitas ${ }^{1}$ \\ Paulo Sérgio Sausen² \\ Airam Tereza Zago Romcy Sausen ${ }^{3}$ \\ Manuel Martin Pérez Reimbold ${ }^{4}$ \\ Programa de Pós-Graduação Stricto Sensu em Modelagem Matemática, UNIJUÍ, Ijuí, RS
}

\begin{abstract}
Resumo. Neste artigo é apresentada uma metodologia alternativa ao Modelo RV, baseada no método de Fourier, para predição do tempo de vida de baterias utilizadas em dispositivos móveis. A metodologia proposta foi avaliada através da comparação entre os resultados das simulações computacionais do modelo e dados experimentais de baterias de Li-Po, sendo que em descargas variáveis obteve um erro médio de 1,55\%.
\end{abstract}

Palavras-chave. Modelo de Difusão, Simulação Computacional, Aplicações em Engenharia, Tempo de Vida de Baterias

\section{Introdução}

A evolução tecnológica das últimas décadas provocou mudanças, tanto nos ambientes e atividades do cotidiano, quanto na forma que nos relacionamos com o mundo. A necessidade de estar conectado com acesso imediato à rede de voz e dados, vem contribuindo para a popularização dos dispositivos móveis - celulares, smartphones, tablets, notebooks, entre outros. Os dispositivos móveis agregam mobilidade, comodidade e facilidade de uso, contudo, têm o tempo de funcionamento limitado pela duração da fonte de energia, ou seja, pelo tempo de vida da bateria.

As baterias recarregáveis utilizadas em dispositivos móveis, têm capacidade finita para armazenamento de energia, necessitando uma recarga a cada período de uso. Deste modo, é importante dispor de métodos para predizer o tempo de vida das baterias e, consequentemente, o tempo de funcionamento dos dispositivos que utilizam-nas como fonte de energia. Uma das formas de realizar esta predição é a partir da utilização da modelagem

\footnotetext{
${ }^{1}$ douglasjoziel@outlook.com

${ }^{2}$ sausen@unijui.edu.br

3 airam@unijui.edu.br

${ }^{4}$ manolo@unijui.edu.br
} 
matemática, que pode descrever o comportamento dinâmico da descarga a partir de suas características físicas reais, ou de um conjunto reduzido de dados experimentais.

A predição do tempo de vida de baterias, empregando a modelagem matemática, permite descrever o processo de descarga das mesmas a partir da simulação de sistemas semelhantes ao real. Ao longo dos anos, diferentes modelos de baterias são desenvolvidos [1]; dentre eles, encontram-se os analíticos, que descrevem a bateria de uma forma abstrata, sendo computacionalmente eficientes e flexíveis, requerendo avaliação de expressões analíticas, que podem ser configuradas para diferentes tipos de baterias [1].

Neste trabalho, é apresentada uma metodologia alternativa para resolução do Modelo de Difusão de Rakhmatov-Vrudhula (RV) [2], para predizer o tempo de vida de vida de baterias de Lítio-Íon Polímero (Li-Po) utilizadas em dispositivos móveis. O principal objetivo do presente trabalho é de agregar ao conhecimento científico, a priori estabelecido, os resultados alcançados através de modelos matemáticos de fácil compreensão e implementação computacional, contribuindo assim com projetistas de baterias, dispositivos móveis e softwares de gestão energética, no desenvolvimento e melhoramento de recursos tecnológicos que ampliem a eficiência energética das baterias.

O artigo está organizado da seguinte forma: na Seção 2 é apresentado o Modelo RV, bem como o desdobramento algébrico, em linhas gerais, da resolução matemática pela metodologia alternativa proposta; na Seção 3 é apresentado o método de estimação dos parâmetros dos modelos, neste caso, utilizando o método dos Mínimos Quadrados (MQ); na Seção 4 são apresentados os resultados das simulações e as análises comparativas com os dados experimentais de baterias de Li-Po; e, por fim, na Seção 5 são apresentadas as considerações finais e as perspectivas para os trabalhos futuros.

\section{Modelagem Matemática}

Nesta seção é apresentado o Modelo RV e, também, a metodologia alternativa proposta. Por definição, baterias são dispositivos que convertem a energia química, armazenada em seus materiais constituintes, em energia elétrica, por meio de uma reação de oxirredução eletroquímica. Na prática, a descarga de uma bateria têm características e efeitos não lineares - nível de cutoff, efeito de recuperação, taxa de capacidade, entre outros - que interferem diretamente no tempo de vida da bateria [3].

O modelo RV descreve o processo de difusão de espécies eletroativas, durante a descarga de uma bateria, com base nas Leis de Fick. Para isso, considera-se a evolução e a concentração dos materiais ativos na bateria durante o processo de descarga, modelando-a como um processo de difusão unidimensional em uma região finita [2]. Matematicamente, o modelo é expresso por meio de duas EDPs, dadas por

$$
\begin{aligned}
& -J(x, t)=D \frac{\partial C(x, t)}{\partial x} \\
& \frac{\partial C(x, t)}{\partial t}=D \frac{\partial^{2} C(x, t)}{\partial x^{2}},
\end{aligned}
$$

onde $J(x, t)$ é o fluxo das espécies eletroativas em função da distância $x \in[0, w]$ do eletrodo e do tempo $t \in[0, L], D$ é a constante de difusão, e $C(x, t)$ é a concentração de espécies. 
Para uma bateria completamente carregada a concentração de espécies é constante através do comprimento do eletrólito, disso decorre a condição inicial

$$
C(x, 0)=C^{*},
$$

onde: $C^{*}$ é a concentração inicial de espécies.

Conforme a Lei de Faraday, o fluxo em uma extremidade do eletrodo é proporcional à corrente de descarga e na outra é nulo. Assim, para descarga de uma corrente $i(t)$ e tempo $0<t<\infty$, temos como condições de fronteira

$$
\begin{aligned}
& \left.\frac{\partial C(x, t)}{\partial x}\right|_{x=0}=\frac{i(t)}{v F A D} \\
& \left.\frac{\partial C(x, t)}{\partial x}\right|_{x=w}=0,
\end{aligned}
$$

onde: $A$ é a área da superfície do eletrodo, $F$ é a constante de Faraday ${ }^{5}$ e $v$ é o número de elétrons envolvidos na reação química na superfície do eletrodo.

Aplicando-se o método da Transformada de Laplace e suas definições [5] e, utilizando a condição inicial e as condições de contorno, tem-se uma resolução para o sistema de EDPs (1) e (2), que relaciona o tempo de vida da bateria $t=L$ e a corrente de descarga $i(t)=I$, expressa por

$$
\alpha=2 I \sqrt{L}\left[1+2 \sum_{m=1}^{10}\left(e^{-\frac{\beta^{2} m^{2}}{L}}-\frac{\pi e^{-\frac{\beta^{2} m^{2}}{L}}}{\pi-1+\sqrt{1+\pi \frac{L}{\beta^{2} m^{2}}}}\right)\right]
$$

onde: $\alpha$ e $\beta$ são parâmetros que dependem do tipo de bateria, sendo estimados a partir de dados experimentais.

\subsection{Modelo RV via Método de Fourier}

Como descrito, a solução do conjunto de equações (1) a (5) do Modelo RV são obtidas aplicando a Transformada de Laplace $[2,3,6]$. Contudo, no intuito de determinar todos os parâmetros iniciais presentes nas equações deste modelo, busca-se encontrar neste trabalho uma solução que, por hipótese, possa ser escrita como

$$
C(x, t)=H(x, t)+V(x, t),
$$

onde: $H(x, t)$ é uma solução particular do sistema, obtida por Tentativa Criteriosa, e $V(x, t)$ é a solução homogênea, obtida analiticamente pelo método de Fourier (i.e., Separação de Variáveis) [7].

\footnotetext{
${ }^{5}$ A constante de Faraday é uma constante física fundamental que representa a carga molar elementar. Atualmente, o Instituto Nacional de Padrões e Tecnologia (NIST) recomenda para a constante de Faraday o valor de $96485,33289 \mathrm{C} \mathrm{mol}^{-1}[4]$.
} 
Considerando, pelo método de Tentativa Criteriosa [7], que a função $H(x, t)$ seja descrita pela expressão dada por

$$
H(x, t)=a x^{2}+b x+c t,
$$

obtém-se, a partir da condição de fronteira (4), que

$$
\left.\frac{\partial H(x, t)}{\partial x}\right|_{x=0}=\left.\frac{\partial C(x, t)}{\partial x}\right|_{x=0} \Rightarrow 2 a(0)+b=\frac{i(t)}{v F A D} \Rightarrow b=\frac{i(t)}{v F A D}
$$

e da condição de fronteira (5), que

$$
\left.\frac{\partial H(x, t)}{\partial x}\right|_{x=w}=\left.\frac{\partial C(x, t)}{\partial x}\right|_{x=w} \Rightarrow 2 a(w)+b=0 \Rightarrow a=-\frac{i(t)}{2 w v F A D}
$$

e da equação (2), que

$$
\frac{\partial H(x, t)}{\partial t}=D \frac{\partial^{2} H(x, t)}{\partial x^{2}} \Rightarrow c=2 D a \Rightarrow c=-\frac{i(t)}{w v F A} .
$$

Substituindo (8) em (2), (3), (4) e (5), com desdobramento algébrico elementar, obtémse facilmente a EDP do problema homogêneo dada por

$$
\frac{\partial V(x, t)}{\partial t}=D \frac{\partial^{2} V(x, t)}{\partial x^{2}}
$$

e a condição inicial

$$
V(x, 0)=C^{*}-H(x, 0)
$$

e, ainda, as condições de fronteira homogêneas

$$
\left.\frac{\partial V(x, t)}{\partial x}\right|_{x=0}=\left.\frac{\partial V(x, t)}{\partial x}\right|_{x=w}=0 .
$$

A solução geral da EDP (12) é dada por

$$
V(x, t)=\frac{1}{2} K_{0}+\sum_{n=1}^{\infty} K_{n} e^{-\frac{n^{2} \pi^{2} D}{w^{2}} t} \cos \left(\frac{n \pi}{w} x\right),
$$

onde: $K_{0}$ e $K_{n}$ são coeficientes de Fourier

$$
K_{0}=2\left(C^{*}-\frac{a}{3} w^{2}-\frac{b}{2} w\right)
$$

e

$$
K_{n}=-\frac{4 a w^{2}}{n^{2} \pi^{2}}(-1)^{n}-\frac{2 b w}{n^{2} \pi^{2}}\left((-1)^{n}-1\right) .
$$


Das equações (8) e (15), substituídas na equação (7), para $i(t)=I$ e $x=0$, tem-se que a solução do conjunto de equações (1) a (5) do Modelo RV pela metodologia proposta é, portanto,

$$
C(0, t)=-\frac{I}{w v F A} t+C^{*}-\frac{I w}{3 v F A D}+\frac{2 I w}{v F A D \pi^{2}} \sum_{n=1}^{\infty} \frac{1}{n^{2}} e^{-\frac{n^{2} \pi^{2} D}{w^{2}} t},
$$

onde: $F$ é a constante de Faraday, $I$ é a corrente de descarga e, $A, C^{*}, D, v$ e $w$ são parâmetros do Modelo RV, a serem determinados a partir de dados experimentais.

\section{Estimação de Parâmetros}

Nesta seção é apresentada a estimação dos parâmetros. Nos ensaios laboratoriais foram utilizadas 8 baterias novas de Li-Po, modelo PL-383562-2C. Os dados experimentais foram adquiridos a partir da plataforma de testes (i.e., testbed), desenvolvida por integrantes do GAIC [8]. Para estimação dos parâmetros, foram utilizados 15 perfis de descarga constante, variando de $75 \mathrm{~mA}$ a $775 \mathrm{~mA}$, com intervalo entre perfis de $25 \mathrm{~mA}$. Cada perfil gerou, de modo individual, um tempo de descarga para cada bateria. A partir destes ensaios, foi possível calcular o tempo de vida médio para cada perfil.

O MQ é um método de otimização que busca encontrar o valor ótimo dos parâmetros para um determinado conjunto de dados, através da minimização da soma dos quadrados dos resíduos. Este método é descrito pela seguinte equação

$$
V_{N}\left(\vec{\zeta}, Z^{N}\right)=\frac{1}{N} \sum_{t=1}^{N}(y(t)-\hat{y}(t \mid \zeta))^{2},
$$

onde: $\vec{\zeta}$ é um vetor que contém os parâmetros a serem estimados, $Z^{N}$ é um vetor que contém as entradas e saídas experimentais, $y(t)$ são as saídas experimentais medidas, $\hat{y}(t \mid \zeta)$ são as saídas calculadas a partir do modelo que contém os parâmetros que se deseja estimar, e $N$ é o número de dados experimentais [9].

As simulações foram implementadas com auxílio do software de computação algébrica e numérica MatLab, em uma rotina para a função de otimização lsqnonlin, que possui internamente a rotina dos MQ para problemas de otimização não linear. Os parâmetros encontrados para o Modelo RV são: $\alpha=2246,1$ e $\beta=3,78$, para resolução original do modelo, e $A=2,5410^{-5}, C^{*}=4,2, D=0,08, v=4591,2$ e $w=1$, para a metodologia proposta.

\section{Resultados das Simulações}

Seguindo a mesma metodologia descrita na Seção 3, a partir de uma série de medições experimentais utilizando um smartphone, definiu-se 8 perfis de descarga realísticos, com variações da corrente ao longo do tempo (cf. Tabela 1). Ao contrário das descargas constantes, esses perfis possuem variações ao longo do tempo, assim, os efeitos não lineares têm maior presença, tornando-se mais fiel ao perfil de utilização de um usuário. 
Tabela 1: Ciclo dos perfis de descarga variável.

\begin{tabular}{ccc}
\hline Perfil & Correntes $(m A)$ & Tempo $(\mathrm{min})$ \\
\hline P1 & $100-10-150-10-100-10-200$ & $5-5-5-5-5-5-10$ \\
P2 & $250-400-50-200-550$ & $10-10-5-15-10$ \\
P3 & $750-450-200-150-250-100$ & $5-10-10-5-5-10$ \\
P4 & $100-200-300-400-500-600-700$ & $10-10-10-10-10-10-10$ \\
P5 & $700-600-500-400-300-200-100$ & $10-10-10-10-10-10-10$ \\
P6 & $170-270-10-140-230-10-270$ & $5-20-30-10-20-10-30$ \\
P7 & $270-10-120-170-10-270-170$ & $5-10-10-15-10-15-5$ \\
P8 & $200-10-300-10-200-10-400$ & $2,5-5-2,5-5-2,5-5-5$ \\
\hline
\end{tabular}

Os resultados obtidos nas simulações foram comparados com um conjunto de dados experimentais, obtidos através da plataforma de testes (cf. Tabela 2). Percebe-se que o Modelo prediz satisfatoriamente o tempo de vida de baterias de Li-Po, sendo que pela metodologia proposta alcançou um resultado ligeiramente melhor, apresentando um erro médio de 1,55\%, em contraste com o erro médio de 1,86\% da solução original. Quando comparado o erro obtido pelo modelo RV com a nova metodologia proposta percebe-se uma melhora de aproximadamente $20 \%$ em termos de acurácia.

Tabela 2: Resultados das simulações.

\begin{tabular}{cccccc}
\hline \multirow{2}{*}{ Perfil } & \multirow{2}{*}{$L_{e}($ min $)$} & \multicolumn{2}{c}{ RV (original) } & \multicolumn{2}{c}{ RV (proposto) } \\
& & $L_{s}(\min )$ & Erro (\%) & $L_{s}(\min )$ & Erro (\%) \\
\hline P1 & 479,68 & 479,38 & 0,06 & 476,08 & 0,75 \\
P2 & 149,38 & 148,53 & 0,57 & 147,77 & 1,08 \\
P3 & 141,76 & 144,17 & 1,70 & 140,85 & 0,64 \\
P4 & 126,62 & 122,9 & 2,94 & 125,97 & 0,51 \\
P5 & 98,51 & 98,35 & 0,16 & 97,68 & 0,85 \\
P6 & 284,94 & 269,48 & 5,43 & 274,93 & 3,51 \\
P7 & 322,01 & 331,55 & 2,96 & 318,08 & 1,22 \\
P8 & 324,17 & 327,58 & 1,05 & 311,75 & 3,83 \\
\hline \multicolumn{5}{c}{$E_{m}=1,86$} \\
\hline
\end{tabular}

\section{Conclusões}

Neste trabalho foi apresentada uma metodologia alternativa de resolução proposta utilizando o método de Fourier, para o Modelo RV. Efetuada a estimação dos parâmetros, foram realizadas simulações computacionais dos modelos e, em seguida, comparados os resultados com um conjunto de dados experimentais. Verificou-se, inicialmente, que para descargas variáveis o Modelo RV original descreveu os dados experimentais com um erro médio de 1,86\%; utilizando, porém, a metodologia proposta o erro médio foi de apenas $1,55 \%$. Destaca-se ainda que para descargas contínuas, o modelo proposto obteve uma melhora de aproximadamente $20 \%$ na predição do tempo de vida das baterias.

A metodologia proposta ao Modelo RV, além do ganho significativo em acurácia, possibilita a estimação de todos os parâmetros iniciais do modelo, ao contrário do modelo 
original que realiza a simplificação dos parâmetros em apenas dois: $\alpha$ e $\beta$. Com isso, a extensão proposta dá aos projetos que envolvem baterias, aporte ao desenvolvimento de novas tecnologias, a partir do estudo sistêmico de todos os parâmetros que compõem o Modelo RV. A otimização do tempo de vida de baterias, neste caso, pode se resumir à análise dos melhores parâmetros para compor um novo tipo de bateria.

Como trabalhos futuros sugere-se estender este estudo à otimização de parâmetros da bateria, para maximização do tempo de vida. Concomitantemente, comparar a metodologia aqui proposta às categorias de outros modelos de bateria, uma vez que o presente estudo ficou restrito aos modelos analíticos.

\section{Referências}

[1] D. J. B. Freitas. Estudo e aplicação de modelos analíticos na predição do tempo de vida de baterias utilizadas em dispositivos móveis: proposição de extensões aos modelos tradicionais. Dissertação de mestrado, Universidade Regional do Noroeste do Estado do Rio Grande do Sul, Ijuí-RS, set. 2015.

[2] D. Rakhmatov and S. Vrudhula. An analytical high-level battery model for use in energy management of portable electronic systems. In Proceedings of the International Conference on Computer Aided Design (ICCAD '01), pages 488-493, Piscataway, 2001. IEEE Press.

[3] M. R. Jorgerden and B. Haverkort. Battery modeling. Technical report, Faculty Electrical Engineering, jan. 2008.

[4] NIST. Fundamental physical constants: Faraday constant. Disponível em: http:// physics.nist.gov/cgi-bin/cuu/Value?f|search_for=faraday. Acesso em: 22 ago. 2015 .

[5] R. D. Strum and J. R. Ward. Equações diferenciais: solução pela transformada de Laplace. Ao Livro Técnico, Rio de Janeiro, 1971.

[6] L. Fransozi. Modelagem matemática do tempo de vida de baterias de lítio íon polímero através de modelos híbridos. Dissertação de mestrado, Universidade Regional do Noroeste do Estado do Rio Grande do Sul, Ijuí-RS, fev. 2015.

[7] R. C. Bassanezi and W. C. F. Junior. Equações diferenciais com aplicações. Harbra, São Paulo, 1988.

[8] H. B. Nonemacher, L. Minelli, P. S. Sausen, and A. Sausen. Desenvolvimento de um testbed para avaliação de modelos matemáticos utilizados na predição do tempo de vida de baterias. In Congresso Regional de Iniciação Científica e Tecnológica em Engenharia, 2010.

[9] L. C. Romio. Modelagem matemática do tempo de vida de baterias utilizando a teoria de Identificação de Sistemas. Dissertação de mestrado, Universidade Regional do Noroeste do Estado do Rio Grande do Sul, Ijuí-RS, mar. 2013. 